\title{
Mucin-producing carcinoma of the gallbladder associated with primary sclerosing cholangitis and ulcerative colitis
}

\author{
Hiroshi Noda $\cdot$ Fumihiro Chiba $\cdot$ Nobuyuki Toyama $\cdot$ \\ Fumio Konishi
}

Received: 10 September 2007/ Accepted: 25 October 2007/Published online: 19 December 2008

(C) Springer 2008

\begin{abstract}
Mucin-producing carcinoma of the gallbladder is very rare. We report here a case of mucin-producing carcinoma of the gallbladder associated with primary sclerosing cholangitis (PSC) and ulcerative colitis (UC). A 74-year-old female had been treated with salazosulfapyridine and ursodesoxycholic acid becase of UC and PSC. After 7 years of treatment, laboratory data showed that the liver function took a turn for the worse, and the patient was admitted to our hospital for further examination. Enhanced computed tomography and ultrasonography showed an enlarged gallbladder associated with wall thickening and diffuse papillary protrusion. Endoscopic retrograde cholangiography showed stenosis and dilatation of the bile duct, which were compatible with PSC. Under the diagnosis of an early carcinoma of the gallbladder, we performed simple cholecystectomy. The tumor showed a papillary growth pattern located diffusely in the gallbladder with a massive amount of mucin filling the gallbladder. Histologically, it was diagnosed as a papillary adenocarcinoma localized in the mucosal layer. To the best of our knowledge, this is the first case of mucin-producing carcinoma of the gallbladder associated with PSC and UC. PSC and UC patients should be regarded as a high-risk group not only for cholangiocarcinoma but also carcinoma of the gallbladder.
\end{abstract}

Keywords Gallbladder carcinoma - Ulcerative colitis . Primary sclerosing cholangitis

H. Noda $(\bowtie) \cdot$ F. Chiba $\cdot$ N. Toyama $\cdot$ F. Konishi Department of Surgery, Saitama Medical Center, Jichi Medical University, 1-847 Amanuma-cho, Omiya-ku, Saitama 330-8503, Japan

e-mail: noda164@omiya.jichi.ac.jp

\section{Introduction}

Mucin-producing carcinoma of the gallbladder is very rare. According to our literature survey, since the first report by Itabashi et al. in 1986 [1], only 29 cases have been reported in the literature [2]. This disease histologically includes two different types of carcinoma: (1) a well-differentiated adenocarcinoma that usually presents with a papillary growth pattern and produces mucin in the gallbladder lumen or (2) a mucinous carcinoma that has a massive mucinous pool within the carcinoma tissues $[2,3]$. We report here a case of the former type of mucinproducing carcinoma of the gallbladder associated with primary sclerosing cholangitis (PSC) and ulcerative colitis (UC).

It is well known that patients suffering from PSC and $\mathrm{UC}$ are at high risk for cholangiocarcinoma. In addition, it has also been reported that the prevalence of gallbladder polyps in PSC patients was $4 \%$, and gallbladder neoplasms in PSC are malignant in approximately $40-60 \%$ of the cases $[4,5]$. Thus, previous reports suggest some correlation among the PSC and UC patients and not only for cholangiocarcinoma but also carcinoma of the gallbladder [4-7]. In this report, we demonstrate the clinicopathological features of a mucin-producing carcinoma of the gallbladder associated with PSC and UC. According to our literature survey, this is the first report of a mucin-producing carcinoma of the gallbladder associated with PSC and UC in the world.

\section{Case report}

A 74-year-old female had been treated with salazosulfapyridine and ursodesoxycholic acid becase of UC and PSC 
for 7 years in a local clinic. In 2006, laboratory data showed that her liver function took a turn for the worse, and ultrasonography demonstrated a diffuse protruding lesion of the gallbladder. Subsequently, she was admitted to our hospital for further examination.

On physical examination, a tender mass was palpated in the right upper abdomen. Ultrasonography demonstrated a diffuse high echoic papillary protrusion in the gallbladder with mild wall thickening (Fig. 1a). Enhanced computed tomography also showed an enlarged gallbladder associated with wall thickening and diffuse papillary protrusion with mild enhancement (Fig. 1b). Endoscopic retrograde cholangiography demonstrated stenosis and dilatation of the bile duct, which were compatible with PSC, but could not visualize the cystic duct and gallbladder (Fig. 1c).

Under the diagnosis of an early carcinoma of the gallbladder, we performed simple cholecystectomy. The tumor showed a papillary growth pattern located diffusely in the gallbladder with a massive mucin pool filling the gallbladder (Fig. 2a). Histologically, it was diagnosed as a papillary adenocarcinoma localized in the mucosal layer (Fig. 2b). In addition, histologically, chronic inflammation was not present in the noncancerous mucosa of the gallbladder. The patient was discharged after an uneventful postoperative period. She has been observed in the outpatient clinic for 1 year without recurrence of carcinoma.

\section{Discussion}

Mucin-producing carcinoma of the gallbladder preponderantly occurs in older women as in our case, and its primary clinical symptoms are mainly right hypochondralgia and jaundice [2, 3]. Histopathologically, as mentioned previously, the mucin-producing carcinoma of the gallbladder includes two different types [2,3], and our case is a type of well-differentiated adenocarcinoma that presents a papillary growth pattern and produces massive amounts of mucin in the gallbladder lumen. Tien et al. [2] previously analyzed the characteristics of radiological findings of mucin-producing carcinoma of the gallbladder and suggested that when a papillary protrusion or thickened wall is seen in the enlarged gallbladder, mucin-producing carcinoma should be included in the differential diagnosis. Radiological findings of our case clearly agreed with these characteristics and supported their suggestions. A mucinproducing carcinoma of our type is potentially less invasive because of its tendency toward intraluminal growth, in contrast to mucinous carcinoma with a tendency toward invasive growth, which is associated with a poor prognosis $[2,3]$. We performed a curative cholecystectomy for the patient, and thus, we may be able to expect a good prognosis.

Previous reports suggest some correlation among the PSC and UC patients and not only for cholangiocarcinoma but also carcinoma of the gallbladder [4-7]. Buckles et al.
Fig. 1 a Ultrasonography and b enhanced computed

tomography showed an enlarged gallbladder associated with wall thickening and diffuse papillary protrusion with mild enhancement, which is compatible with a mucinproducing carcinoma of the gallbladder. c Endoscopic retrograde cholangiography demonstrated stenosis and dilatation of the bile duct, which were compatible with PSC
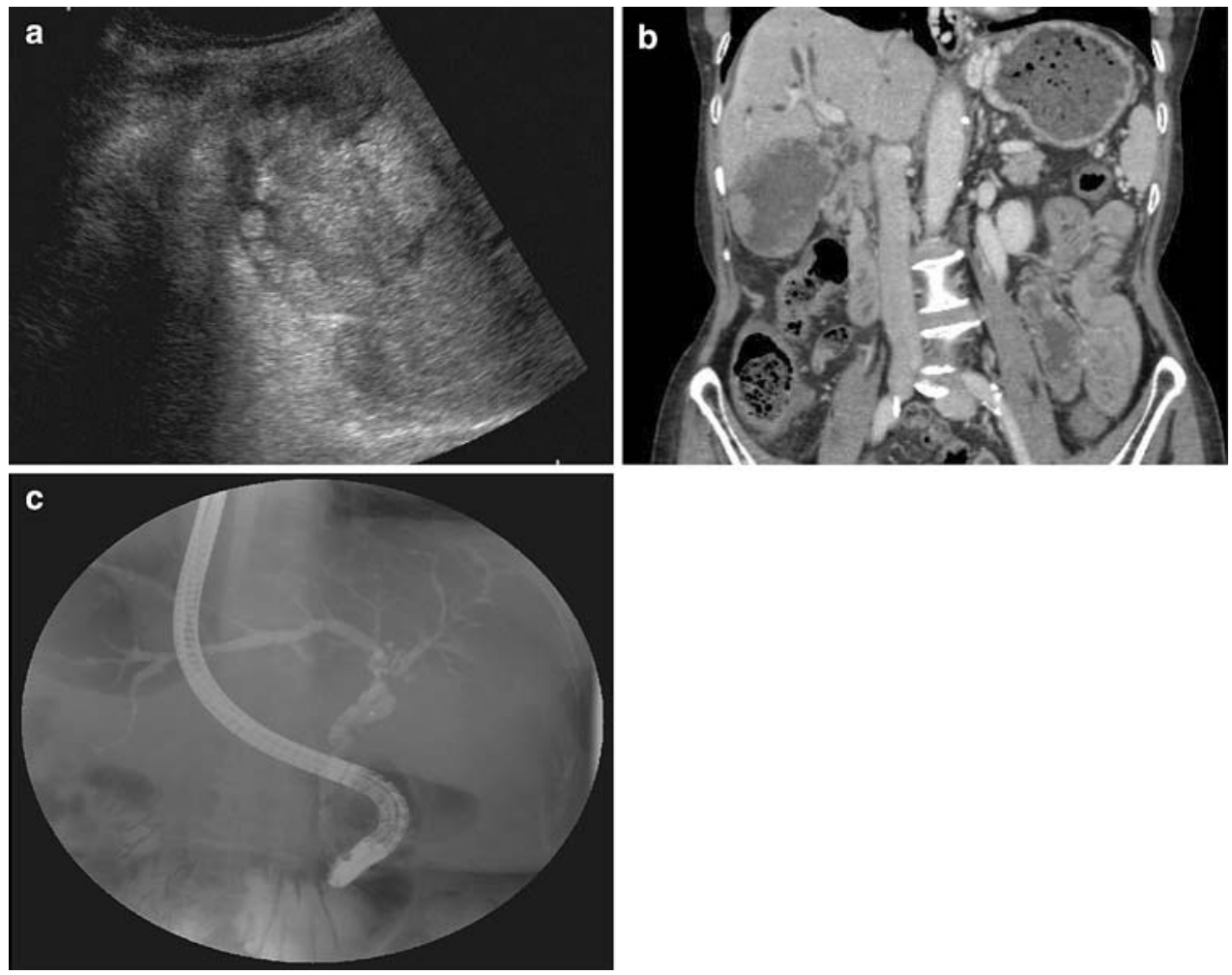


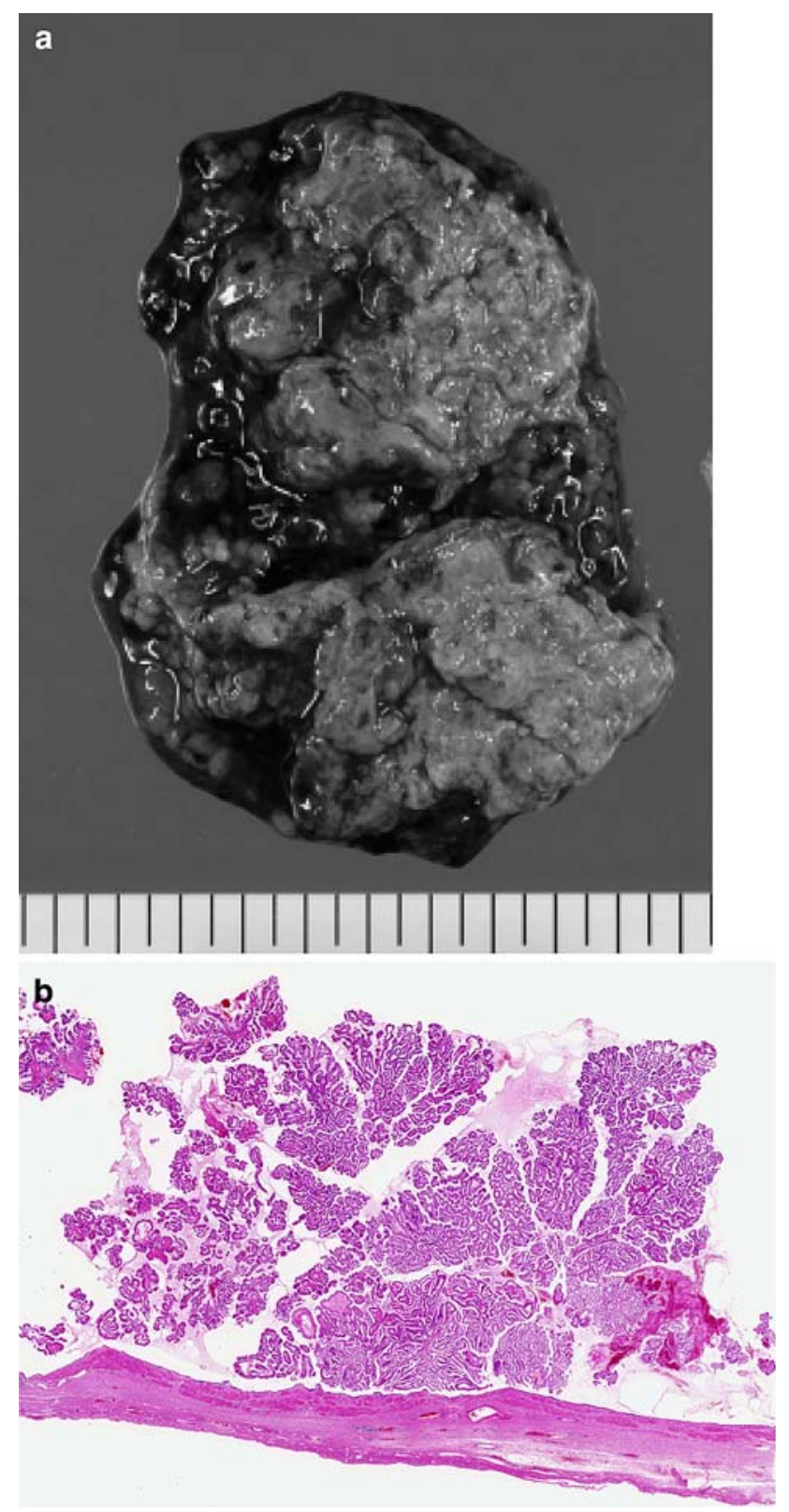

Fig. 2 a The tumor showed a papillary growth pattern located diffusely in the gallbladder with a massive amount of mucin filling the gallbladder. b Histologically, the tumor was diagnosed as a papillary adenocarcinoma localized in the mucosal layer

[5] suggested that the chronically inflamed gallbladder mucosa in PSC patients, like the inflamed colon in ulcerative colitis patients, may be associated with risk of malignant degeneration. Carcinoma of the gallbladder is a malignancy with a poor prognosis because of its invasive nature and the difficulty of detecting it at an early stage [8]. Ultrasonography is still the most simple and cost-effective manner of evaluating the gallbladder, and in our case the tumor of the gallbladder was detected first by ultrasonography. Given the increased risk of developing cholangiocarcinoma and carcinoma of the gallbladder for patients with PSC and UC, it is acceptable to periodically assess the biliary tract in this population with ultrasonography. Early detection may contribute to a good prognosis for the patients.

In conclusion, we experienced a mucin-producing carcinoma of the gallbladder associated with PSC and UC. Assessing the biliary tract, not only for cholangiocarcinoma but also carcinoma of the gallbladder, with repeated radiographic examinations for PSC and UC patients is advocated. In addition, it is worthy to note that when a papillary protrusion or thickened wall is seen in the enlarged gallbladder as in our case, mucin-producing carcinoma should be included in the differential diagnosis.

\section{References}

1. Ishibashi H, Hachisuka K, Yamaguchi A, Isotani M, Fukada S, Kato J, et al. A case of mucin-producing carcinoma of the gallbladder (in Japanese). Tan to Sui. 1986;7:1173-8.

2. Tian H, Matsumoto S, Takaki H, Kiyosue H, Komatsu E, Okino Y, et al. Mucin-producing carcinoma of the gallbladder: imaging demonstration in four cases. J Comput Assist Tomogr. 2003;27: $150-4$.

3. Levy AD, Murkata LA, Rohrmann CA. Gallbladder carcinoma: radiologic-pathologic correlation. Radiographics. 2001;21:295314.

4. Brandt DJ, MacCarty RL, Charboneau JW, LaRusso NF, Weisner RH, Ludwig J. Gallbladder disease in patients with primary sclerosing cholangitis. Am J Roentogenol. 1998;150:571-4.

5. Buckel DC, Lindor KD, LaRusso NF, Petrovic LM, Gores GJ. In primary sclerosing cholangitis, gallbladder polyps are frequently malignant. Am J Gastroenterol. 2002;97:1138-42.

6. Yamamoto T, Uki K, Takeuchi K, Nagashima N, Honjo H, Sakurai $\mathrm{N}$, et al. Early gallbladder carcinoma associated with primary sclerosing cholangitis and ulcerative colitis. J Gastroenterol. 2003;38:704-6.

7. Kobayashi H, Hosotani R, Imai Y, Wada K, Kajiwara T. Primary sclerosing cholangitis in which differential diagnosis from gallbladder carcinoma was difficult. J Hepatobiliary Pancreat Surg. 2005;12:151-4.

8. Donohue JH. Present status of the diagnosis and treatment of gallbladder carcinoma. J Hepatobiliary Pancreat Surg. 2001;8: $530-4$. 